\title{
Optimal Award Scheme in Innovation Tournaments
}

\author{
Laurence Ales \\ Tepper School of Business, Carnegie Mellon University, Pittsburgh, PA 15213, ales@cmu.edu \\ Soo-Haeng Cho \\ Tepper School of Business, Carnegie Mellon University, Pittsburgh, PA 15213, soohaeng@andrew.cmu.edu \\ Ersin Körpeoğlu \\ School of Management, University College London, London, UK, E14 5AB, e.korpeoglu@ucl.ac.uk \\ Forthcoming in Operations Research
}

\begin{abstract}
In an innovation tournament, an organizer solicits innovative ideas from a number of independent agents. Agents exert effort to develop their solutions, but their outcomes are unknown due to technical uncertainty and/or subjective evaluation criteria. In order to incentivize agents to make their best effort, the organizer needs to devise a proper award scheme. While extant literature either assumes a winner-take-all scheme a priori or shows its optimality under specific distributions for uncertainty, this paper derives necessary and sufficient conditions under which the winner-take-all scheme is optimal. These conditions are violated when agents perceive it very likely that only few agents receive high evaluation or when a tournament does not require substantial increase in agents' marginal cost of effort to develop high-quality solutions. Yet, the winner-take-all scheme is optimal in many practical situations, especially when agents have symmetric beliefs about their evaluation. In this case, the organizer should offer a larger winner prize when he is interested in obtaining a higher number of good solutions, but interestingly the organizer need not necessarily raise the winner prize when anticipating more participants to a tournament.
\end{abstract}

Subject classifications: Games/group decisions: Tournament; Research and development: Innovation Area of review: Operations and Supply Chains

\section{Introduction}

In an innovation tournament (also called a contest), a tournament organizer seeks solutions to an innovation-related problem from a number of independent agents. Before a tournament begins, the organizer announces an award scheme and a set of evaluation criteria. Then agents who are interested in participating in the tournament develop and submit their solutions to the problem. After collecting all submissions, the organizer evaluates them and awards the best submissions.

Today a tournament approach has gained popularity in many industries, ranging from high-tech industries (e.g., DARPA Grand Challenge in which participants develop autonomous vehicles) to innovative software development (e.g., Samsung Smart App Challenge in which software developers submit apps for Samsung's mobile products). Companies organize innovation tournaments by 
themselves or they use tournament platforms such as Ennomotive and InnoCentive which intermediate thousands of innovation tournaments every year for various organizations. A primary motivation to use a tournament approach, instead of traditional in-house development or contracting with select suppliers, is that a company can potentially collect many diverse solutions to its problem from a large number of experts outside its boundary or ecosystem. This approach may be appealing especially when a company is uncertain about how to create innovative solutions and what constitutes good solutions. From agents' perspective, a tournament is attractive when they anticipate high chances of winning a prize and when a winner's prize is high enough to justify their investment in effort. In order to elicit participation and best effort from agents, therefore, choosing the right award scheme is foremost important to a tournament organizer.

The objective of this paper is to offer insights into how a tournament organizer should compensate participants. Our first research question is when an organizer should grant the entire prize to the best submission (i.e., select a winner-take-all (WTA) award scheme) and when he should not. For this question, prior literature assumes the WTA scheme a priori (e.g., Taylor 1995, Fullerton and McAfee 1999, Kim and Lim 2015, Ales et al. 2016), proves its optimality when uncertainty in agents' solution quality is modeled with logistic or uniform distribution (Kalra and Shi 2001), or shows that it outperforms an alternative scheme that awards the winner and/or the runner-up under Gumbel distribution (Terwiesch and Xu 2008). ${ }^{1}$ Yet, in practice, we observe both WTA and multiple-prize tournaments. For example, many innovation tournaments on engineering problems at Ennomotive and reduction-to-practice (RTP) challenges (which require submission of validated solutions, either in the form of original data or physical samples for technical problems) at InnoCentive adopt the WTA scheme (Ennomotive 2016, InnoCentive 2016b). In contrast, a number of ideation challenges (for producing breakthrough ideas) at InnoCentive awards multiple prizes (InnoCentive 2016a). Also, Samsung Apps Super Apps category in Samsung Smart App Challenge 2012 featured multiple prizes based on the number of downloads of submitted apps (Samsung 2012).

To answer this question, we develop a general model of an innovation tournament that takes into account the following two sources of uncertainty: (i) technical uncertainty due to an agent's search process for the best solution from a number of trials (e.g., a logo designer participating in a logo design tournament for FIFA 2014 World Cup may draw multiple sketches until she chooses the best one to submit), and/or (ii) taste uncertainty due to a subjective or unknown taste of the organizer or other people who evaluate agents' solutions (e.g., it is difficult for a logo designer to predict the specific design of a logo that the organizer would prefer). Whereas existing models consider either type of uncertainty under specific distributions, our model accounts for both types of uncertainty under a general distribution for aggregate uncertainty. This generality not only improves the model's relevance to innovation tournaments in practice (in which both types of 
uncertainty are often co-present and agents can exhibit a variety of beliefs on the subjective taste of the organizer), but also helps reconcile theory with practice. From our analysis, we derive necessary and sufficient conditions under which the WTA scheme is optimal; it turns out that a type of a distribution plays a crucial role in determining the optimality of the WTA scheme. When any of the necessary and sufficient conditions is violated, thus, the WTA scheme is not optimal. For example, in a tournament wherein submitted solutions are evaluated based on popularity among consumers (e.g., the 2012 Samsung Smart App Challenge), an organizer may not choose the WTA scheme. In contrast, when agents have roughly equal beliefs about the chances of getting more positive or negative evaluations than they deserve, the WTA scheme is optimal. More generally, we establish the optimality of the WTA scheme under a broad class of distributions for agents' uncertainty.

We next examine how large a winner prize should be under the WTA scheme. For this question, we first establish an intuitive result that an organizer should offer a larger winner prize when he is interested in obtaining a higher number of good solutions. Similarly, one might expect that an organizer should also raise the winner prize when anticipating more participants to a tournament. Yet, existing theory suggests that the winner prize should be independent of the number of participants: Terwiesch and $\mathrm{Xu}$ (2008) derive this result assuming that each agent's output is the sum of a logarithmic function of effort and an uncertain shock that follows Gumbel distribution. In contrast, our paper shows that an optimal winner prize can be constant, increasing, or counter-intuitively decreasing with the number of participants when modeling an agent's output as the sum of a general increasing, concave function of effort and an uncertain shock that follows a general distribution. A primary reason for this result is that the concavity of the effort function determines agents' marginal returns from additional effort, and hence it affects agents' incentives to make effort in response to increased competition. Our result suggests that the organizer should increase the winner prize with more participants in a simple ideation project, in which generating an average-quality idea for a simple problem requires relatively low effort, yet additional effort does not improve the quality of the idea much; but the organizer may not do so in a tournament that requires agents to exert consistent and significant effort (i.e., slowly decreasing marginal returns from additional effort).

\section{Model and Preliminaries}

Our model adopts the following framework that has been commonly used in the tournament literature. A tournament organizer ("he") solicits innovative solutions to a specified problem from a set of agents ("she"). The organizer first announces how participants of the tournament will be compensated. Then agents decide whether to participate in the tournament, and if they do, they exert effort to develop their solutions, and submit them to the organizer. Finally, the organizer evaluates these solutions and compensates agents according to the announced rule. 
The Model of Agents. Let $\mathcal{N}$ denote a set of agents who can participate in the tournament, and $N=|\mathcal{N}|(\geq 2)$ denote the number of agents in this set. ${ }^{2}$ Each participating agent $i(\in \mathcal{N})$ develops a solution to the problem posed by the organizer, and generates an output $y_{i}(\in \mathcal{Y} \subseteq \mathbb{R} \cup\{-\infty, \infty\})$, which represents the quality of the solution or its monetary benefit to the tournament organizer. The output $y_{i}$ is determined by three components: (i) agent $i$ 's effort, (ii) outcomes of agent $i$ 's random trials and experiments, and (iii) taste of the organizer or other people who evaluate the agent's solution. We elaborate each of these components next.

First, each agent can enhance her output by investing "improvement effort" $q_{i} \in[0, \bar{q}]$. For example, conducting a thorough patent search and literature review will certainly improve agents' outputs (Terwiesch and Xu 2008). Effort $q_{i}$ leads to a deterministic improvement $v\left(q_{i}\right)$ of the output, where $v$ is an increasing and concave function of $q_{i}$.

Second, to generate innovative solutions, each agent may engage in a trial-and-error process by conducting several experiments. Most innovation processes include a concept testing process in which an agent generates potential ideas and selects the best of them (e.g., Dahan and Mendelson 2001). In this process, each agent $i$ determines the number of trials $m_{i}$ (hereafter "trial effort"). In each trial $t\left(=1,2, \ldots, m_{i}\right)$, the agent faces uncertainty in the outcome of a trial which is modeled by a trial shock $\widetilde{\epsilon}_{i t}$. For example, a logo designer may draw multiple sketches until she chooses the best one to submit. Following the convention of Terwiesch and Xu (2008), based on Dahan and Mendelson (2001), we consider the specific case in which trial shocks $\widetilde{\epsilon}_{i t}$ 's are independent random variables that follow a Gumbel distribution with $E\left[\widetilde{\epsilon}_{i t}\right]=0$ and scale parameter $\mu$. The Gumbel distribution is the asymptotic distribution for the maximum of multiple draws from exponentialtailed distributions such as the normal distribution (Dahan and Mendelson 2001). Each agent $i$ observes the realization of $\left(\widetilde{\epsilon}_{i 1}, \widetilde{\epsilon}_{i 2}, \ldots, \widetilde{\epsilon}_{i m_{i}}\right)$ before submitting her solution to the organizer, and selects the best one as her solution. The main difference between the improvement effort and the trial effort is that an agent knows the value of her improvement effort a priori, whereas she is uncertain about the final outcome of the trial effort until the end of the development process.

Third, the output of each agent $i$ is often subject to the taste of its evaluators, which we model by a taste shock $\widetilde{\varepsilon}_{i}$. For example, it is difficult for a logo designer to predict the specific design of a logo the organizer would prefer. Following the literature, we assume that $\widetilde{\varepsilon}_{i}$ 's are independent and identically distributed (i.i.d.) random variables with $E\left[\widetilde{\varepsilon}_{i}\right]=0$. We use a general distribution for $\widetilde{\varepsilon}_{i}$ to model a variety of the agent's belief on unknown taste. This is different from the literature which assume uniform or logistic distributions (Kalra and Shi 2001) or Gumbel distribution (Terwiesch and $\mathrm{Xu} 2008$ ). Unlike trial shocks $\widetilde{\epsilon}_{i t}$ 's, each agent $i$ is uncertain about the taste shock $\widetilde{\varepsilon}_{i}$ even after the development process is over. 
Combining the three components discussed above, we represent the output of agent $i$ as a function of improvement effort $q_{i}$, trial effort $m_{i}$, trial shock $\left(\widetilde{\epsilon}_{i 1}, \ldots, \widetilde{\epsilon}_{i m_{i}}\right)$, and taste shock $\widetilde{\varepsilon}_{i}$, as follows:

$$
y\left(q_{i}, m_{i}, \widetilde{\epsilon}_{i 1}, \ldots, \widetilde{\epsilon}_{i m_{i}}, \widetilde{\varepsilon}_{i}\right)=v\left(q_{i}\right)+\max \left\{\widetilde{\epsilon}_{i t}, t=1, \ldots, m_{i}\right\}+\widetilde{\varepsilon}_{i} .
$$

This general output function takes existing models in the literature as special cases: (i) trial-anderror projects in Terwiesch and $\mathrm{Xu}$ (2008) which assume fixed improvement effort (i.e., $v\left(q_{i}\right)=\gamma$ for some constant $\gamma$ ) and no taste shock (i.e., $\widetilde{\varepsilon}_{i}=0$ with probability 1 ); (ii) ideation projects in Terwiesch and $\mathrm{Xu}(2008)$ which assume no trial-and-error experiments (i.e., $\widetilde{\epsilon}_{i t}=0$ with probability 1) and a specific distribution of the taste shock $\widetilde{\varepsilon}_{i}$ (i.e., Gumbel $\widetilde{\varepsilon}_{i}$ ); and (iii) Ales et al. (2016) which assume no trial-and-error experiments (i.e., $\widetilde{\epsilon}_{i t}=0$ with probability 1 ).

The utility of agent $i, U_{a}\left(q_{i}, m_{i}, x_{i}\right): \mathbb{R}_{+}^{3} \rightarrow \mathbb{R}$, is defined over her improvement effort $q_{i}$, her trial effort $m_{i}$, and the monetary compensation $x_{i}$ that she receives from the organizer. The utility of the agent takes the following form: $U_{a}\left(q_{i}, m_{i}, x_{i}\right)=x_{i}-\psi\left(c_{1} q_{i}+c_{2} m_{i}\right)$, where $c_{1}>0, c_{2}>0$, and $\psi$ is convex and increasing with $\psi(0)=0$. We can interpret this function as follows. When agent $i$ exercises improvement effort $q_{i}$ and trial effort $m_{i}$, her total effort is $e_{i}=c_{1} q_{i}+c_{2} m_{i}$; for example, if $e_{i}$ represents the total labor hours agent $i$ spends, $c_{1}$ and $c_{2}$ are the time it takes for the agent to make one unit of improvement and trial effort, respectively. The agent's disutility or cost associated with the total effort is $\psi\left(e_{i}\right)=\psi\left(c_{1} q_{i}+c_{2} m_{i}\right)$.

We next present a lemma which shows that output function $y$ given in (1) can be simplified to a new function that depends only on agent $i$ 's total effort $e_{i}$ and aggregate shock $\widetilde{\xi}_{i}$. All proofs are presented in Appendix.

Lemma 1. The output function in (1) can be simplified to $y\left(e_{i}, \widetilde{\xi}_{i}\right)=r\left(e_{i}\right)+\widetilde{\xi}_{i}$ in which $e_{i}$ is the total effort, $r$ is a concave and increasing function, and $\widetilde{\xi}_{i}$ is a random shock that is independent of $e_{i}$. For example, if $v\left(q_{i}\right)=\kappa \log \left(q_{i}\right)$ for some $\kappa>0$, then $r\left(e_{i}\right)=\gamma+\theta \log \left(e_{i}\right)$ where $\theta(>0)$ and $\gamma$ are constants.

In the rest of this paper, we will use the simplified output function $y\left(e_{i}, \widetilde{\xi}_{i}\right)=r\left(e_{i}\right)+\widetilde{\xi}_{i}$, and refer to $e_{i}$ as agent $i$ 's "effort" and $\widetilde{\xi}_{i}$ as her "output shock." The output shock $\widetilde{\xi}_{i}(\in \Xi)$ follows cumulative distribution $H$ and density $h$ with $E\left[\widetilde{\xi}_{i}\right]=0$ and support $\Xi=[\underline{s}, \bar{s}]$ where $\bar{s} \in \mathbb{R} \cup\{-\infty\}$ and $\bar{s} \in \mathbb{R} \cup\{\infty\}$. As shown in the proof of Lemma $1, \widetilde{\xi}_{i}$ is equal to $\widetilde{\epsilon}_{i}+\widetilde{\varepsilon}_{i}$, where "technical shock" $\widetilde{\epsilon}_{i} \equiv \max \left\{\widetilde{\epsilon}_{i t}, t=1,2, \ldots, m_{i}\right\}-\mu \log m_{i}$ follows a Gumbel distribution with $E\left[\widetilde{\epsilon}_{i}\right]=0$ and scale parameter $\mu$. We will interpret our results in terms of primitive technical shock $\widetilde{\epsilon}_{i}$ and taste shock $\widetilde{\varepsilon}_{i}$ as well as aggregate output shock $\widetilde{\xi}_{i}$. To analyze the impact of a change in the variance of a general distribution $H(s)$, we use the following notion of a scale transformation (e.g., Rothschild and Stiglitz 1970): Two distribution functions $H_{1}(\cdot)$ and $H_{2}(\cdot)$ differ by a scale transformation if 
there exists parameter $\alpha$ such that $H_{1}(s)=H_{2}(\alpha s)$ for all $s \in \Xi$. The transformed output shock $\widehat{\xi}_{i}=\alpha \widetilde{\xi}_{i}$ of the output shock $\widetilde{\xi}_{i}$ has a mean of 0 and a variance of $\alpha^{2} \operatorname{Var}\left(\widetilde{\xi}_{i}\right)$. Thus, $\widehat{\xi}_{i}$ has a larger variance than $\widetilde{\xi}_{i}$ when $\alpha>1$.

The Model of the Organizer. The organizer compensates agents based on the rank of their outputs. Specifically, the organizer determines a vector of $N$ prizes (awards) referred to as "award scheme," denoted by $\left(A_{(1)}, A_{(2)}, \ldots, A_{(N)}\right)$, such that the agent who produces the $j$-th best output receives a prize of $A_{(j)}$. We refer to the agent who produces the best output as the "winner" of the tournament, and the award scheme that grants the entire prize to the winner (i.e., $A_{(1)}>0$ and $A_{(j)}=0$ for all $j>1$ ) as the winner-take-all (WTA) award scheme.

The organizer maximizes his profit $\Pi_{o}$ from the tournament, where $\Pi_{o}$ is defined over his payoff from the tournament less total prize paid to agents. As in Ales et al. (2016), we consider the general model where the organizer benefits from $K(\in\{1,2, \ldots, N\})$ best outputs, ${ }^{3}$ and refer to those agents who produce the $K$ best outputs as "contributors." Letting $y_{(j)}$ denote the $j$-th best output among $N$ outputs, with $K$ contributors, the organizer's profit can be expressed as:

$$
\Pi_{o}\left(\left(y_{(1)}, y_{(2)}, \ldots, y_{(K)}\right),\left(A_{(1)}, A_{(2)}, \ldots, A_{(N)}\right)\right)=\sum_{j=1}^{K} y_{(j)}-\sum_{j=1}^{N} A_{(j)} .
$$

We now present the organizer's problem that maximizes $\Pi_{o}$ given in $(2)$. Let $\widetilde{\xi}_{(j)}^{N}$ be a random variable with cumulative distribution $H_{(j)}^{N}$ and density $h_{(j)}^{N}$ that represents the $j$-th highest value among $N$ i.i.d. output shocks. Similarly, let $P_{(j)}^{N}\left[e_{i}, e^{*}\right]$ be the probability that the output of agent $i$ is the $j$-th highest output when she exerts effort $e_{i}$ and all other $(N-1)$ agents exert the equilibrium effort $e^{*}$. We can compute this probability as

$$
P_{(j)}^{N}\left[e_{i}, e^{*}\right]=\int_{s \in \Xi} \frac{(N-1) !}{(j-1) !(N-j) !} H\left(s+r\left(e_{i}\right)-r\left(e^{*}\right)\right)^{N-j}\left(1-H\left(s+r\left(e_{i}\right)-r\left(e^{*}\right)\right)\right)^{j-1} h(s) d s .
$$

The organizer solves the following program:

$$
\begin{aligned}
\max _{\left(A_{(1)}, A_{(2)}, \ldots, A_{(N)}\right)} & \Pi_{o}=K r\left(e^{*}\right)+E\left[\sum_{j=1}^{K} \widetilde{\xi}_{(j)}^{N}\right]-\sum_{j=1}^{N} A_{(j)} \\
\text { s.t. } & \frac{1}{N} \sum_{j=1}^{N} A_{(j)} \geq \psi\left(e^{*}\right) \\
& e^{*}=\arg \max _{e \in \mathbb{R}_{+}} \sum_{j=1}^{N} P_{(j)}^{N}\left[e, e^{*}\right] A_{(j)}-\psi(e) .
\end{aligned}
$$

The objective of the organizer given in (4) is to choose $\left(A_{(1)}, A_{(2)}, \ldots, A_{(N)}\right)$ that maximize his expected profit. Participation constraint (5) guarantees that agents receive non-negative utility from the tournament in equilibrium. Constraint (6) is the incentive compatibility constraint that incorporates the agent's utility maximization problem into the organizer's problem. In this problem, 
each agent chooses the effort $e$ that maximizes her expected prize $\sum_{j=1}^{N} P_{(j)}^{N}\left[e, e^{*}\right] A_{(j)}$ less her cost of exerting effort $e, \psi(e)$, assuming that every other agent will choose $e^{*}$ in equilibrium. ${ }^{4}$

\section{Analysis}

In this section, we analyze an optimal award scheme. Let $A=\sum_{j=1}^{N} A_{(j)}$ be the total amount of prizes awarded. For any given $A$, an award scheme $\left(A_{(1)}, A_{(2)}, \ldots, A_{(N)}\right)$ maximizes the organizer's profit $\Pi_{o}$ when it maximizes the first term in (4) by inducing agents to make their best effort in equilibrium, because the second and third terms in (4) are constant. Note from the agent's problem given in (6) that the marginal benefit of additional effort, $\sum_{j=1}^{N}\left(P_{(j)}^{N}\left[e, e^{*}\right]\right)^{\prime} A_{(j)}$, depends on the award scheme $\left(A_{(1)}, A_{(2)}, \ldots, A_{(N)}\right)$, while its marginal cost $\psi^{\prime}(e)$ is independent of this scheme. Thus, the award scheme that maximizes agents' marginal benefit of additional effort induces agents to make their best effort in equilibrium. The following proposition provides a necessary and sufficient condition under which the WTA scheme is optimal.

Proposition 1. For any $A$, it is optimal for the organizer to grant the entire prize to the winner, i.e., $A_{(1)}=A$ and $A_{(j)}=0$ for all $j \in\{2, \ldots, N\}$ if and only if (5) holds and

$$
\left.\frac{\partial P_{(1)}^{N}\left[e, e^{*}\right]}{\partial e}\right|_{e=e^{*}} \geq\left.\frac{\partial P_{(j)}^{N}\left[e, e^{*}\right]}{\partial e}\right|_{e=e^{*}} \text { for all } j>1 \text {. }
$$

Proposition 1 states that the WTA award scheme maximizes the agent's marginal benefit of effort under condition (7). This condition is satisfied when a marginal increase of her effort raises her probability of becoming the winner $\left(P_{(1)}^{N}\left[e, e^{*}\right]\right)$ more than her probability of attaining any other rank $j(>1)\left(P_{(j)}^{N}\left[e, e^{*}\right]\right)$. If condition $(7)$ is violated, then the increased effort of an agent raises the probability of attaining rank $j(>1)$ more than the probability of becoming the winner; and thus the organizer is better off by choosing an award scheme with $A_{(j)}>0$ rather than the WTA scheme. It is important to note that condition (7), hence the optimality of the WTA scheme, depends crucially on the characteristics of the output shock $\widetilde{\xi}_{i}$. This can be seen from the definition of $P_{(j)}^{N}$ given in (3) that shows its dependence on the density $h$ of $\widetilde{\xi}_{i}$.

An important implication of Proposition 1 is that the WTA scheme is not optimal whenever any of the necessary and sufficient conditions presented in the proposition is violated. Specifically, the WTA scheme is not optimal when: (i) the density $h$ of $\widetilde{\xi}_{i}$ violates condition (7) in Proposition 1 , or (ii) agents' participation in a tournament is not guaranteed (i.e., (5) does not hold) under this award scheme. The following proposition provides sufficient conditions for each of these situations.

Proposition 2. For any given A, the winner-takes-all (WTA) award scheme is suboptimal when one of the following conditions is satisfied:

(i) $\lim _{s \rightarrow \bar{s}} h(s)=0, \lim _{s \rightarrow \bar{s}}\left|\frac{h^{\prime}(s)}{h(s)}\right|<\infty$, and

$$
\int_{s \in \Xi}\left[H_{(j)}^{N}(s)-H_{(1)}^{N}(s)\right]\left(\frac{h^{\prime}(s)}{h(s)}\right)^{\prime} d s>0
$$




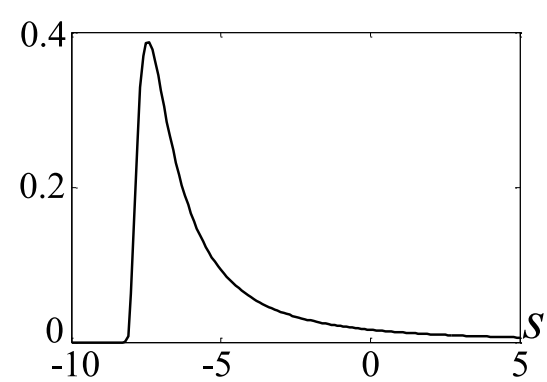

(a)

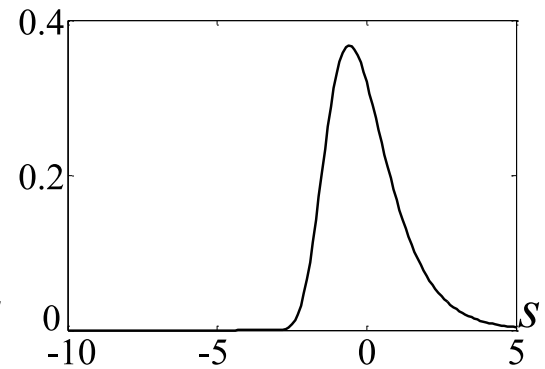

(b)

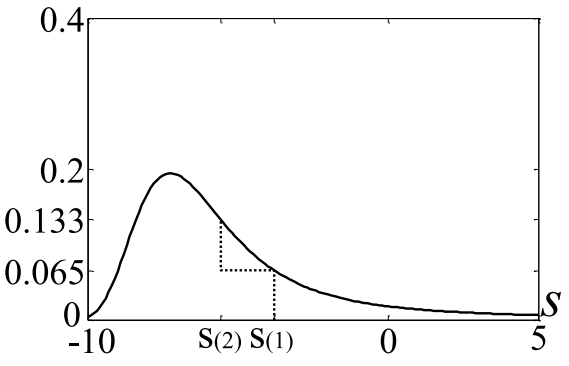

(c)

Figure 1 (a) Frechet density with mean 0, shape parameter $\beta=1.2$, and scale parameter $\mu=1.5$; (b) Gumbel density with mean 0 and scale parameter $\mu=1$; and (c) The convolution of the densities in (a) and (b), where $s_{(j)}$ satisfies $h\left(s_{(j)}\right)=E\left[h\left(\widetilde{\xi}_{(j)}^{N-1}\right)\right]$.

where (8) holds if $h(s)$ is strictly log-convex (i.e., $\left.d^{2} \log h(s) / d s^{2}>0 \forall s\right)$.

(ii) $\frac{A}{N}-\psi\left(\left(\frac{\psi^{\prime}}{r^{\prime}}\right)^{-1}\left(A \int_{s \in \Xi}(N-1) H(s)^{N-2} h(s)^{2} d s\right)\right)<0$.

We first discuss condition (i) using an example in Figure 1. Observe that the density $h$ shown in Figure 1(a) or Figure 1(c) features a large region that is highly convex (which guarantees (8)) and decreasing between its peak point and its fat right tail (which ensures $\lim _{s \rightarrow \bar{s}} h(s)=0$ and $\left.\lim _{s \rightarrow \bar{s}}\left|h^{\prime}(s) / h(s)\right|<\infty\right) .{ }^{5}$ The output shock $\widetilde{\xi}_{i}$ can have the density $h$ shown in Figure 1(a) when the taste shock $\widetilde{\varepsilon}_{i}$ has this density and the technical shock $\widetilde{\epsilon}_{i}=0$ with probability 1 as in ideation projects; or it can have the density $h$ shown in Figure $1(\mathrm{c})$ when the taste shock $\widetilde{\varepsilon}_{i}$ has the density shown in Figure 1(a) and the technical shock $\widetilde{\epsilon}_{i}$ features the Gumbel density shown in Figure 1(b). In these examples, due to the large, highly convex, and decreasing region of $h(s)$, a marginal increase of an agent's effort could increase her probability of attaining some rank $j(>1)$ more than that of becoming the winner. ${ }^{6}$ In practice, this may represent a situation where agents perceive it very likely that only few agents receive high evaluation while most of them receive low evaluation. For example, this may be the case in tournaments wherein agents' outputs are evaluated based on popularity among consumers and only few solutions are expected to be extremely popular (e.g., the number of downloads for apps in the 2012 Samsung Smart App Challenge). It is well known that such herding behavior can cause an upward bias in the evaluation of highly popular solutions (e.g., Li and Hitt 2008, Cha et al. 2007). Similarly, according to Dahan and Mendelson (2001), when a product has a potential for "mega-hits," a fat-tailed distribution like the Frechet distribution shown in Figure 1(a) is a good fit for modeling its profit uncertainty.

Condition (ii) specifies when agents' participation becomes an issue in a tournament under the WTA scheme. The solution to the agent's problem in (6) does not satisfy the participation constraint (5) when each agent's effort in equilibrium (hence, the right hand side of (5)) is too high to be justified by the expected winner prize they may receive (i.e., the left hand side of (5)). In this case, there will be no equilibrium under the WTA award scheme, and hence the WTA scheme 


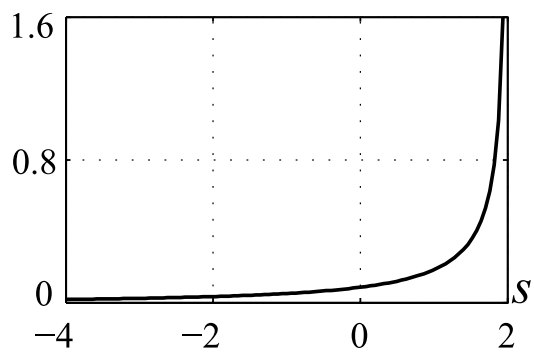

(a)

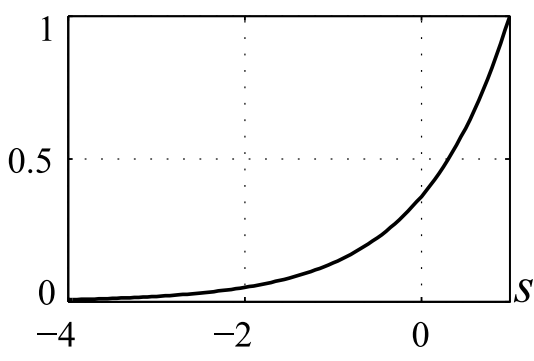

(b)

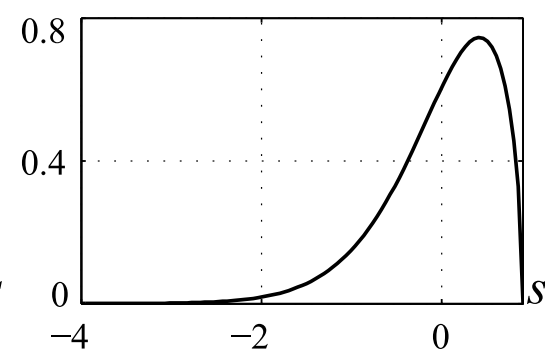

(c)

Figure 2 Weibull density with mean 0 , scale parameter $\mu=1$, and shape parameter $\beta$ : (a) Strictly log-convex $(\beta=0.5)$, (b) log-linear $(\beta=1)$, and (c) strictly log-concave $(\beta=1.5)$.

cannot be optimal to the organizer. Thus, the organizer may award multiple prizes in order to induce agents to participate in a tournament, although they exert lower effort than the effort level under the WTA scheme. In Lemma EC.2 of Online Appendix, we show that condition (ii) holds when agents face a sufficiently low level of uncertainty and/or when the cost function $\psi$ of agents is not highly convex. This suggests that, ceteris paribus, the WTA scheme is less likely to be optimal in a tournament that contains less technical uncertainty while demanding less substantial increase in agents' marginal costs of effort (e.g., ideation tournaments at InnoCentive).

Next, we derive sufficient conditions for $\widetilde{\xi}_{i}$ to satisfy condition $(7)$ under which the WTA scheme is optimal.

Proposition 3. Suppose that (5) holds under the WTA award scheme. Then, for any given A, the WTA award scheme is optimal when the density $h(s)$ of the output shock $\widetilde{\xi}_{i}$ is log-concave (i.e., $\left.d^{2} \log h(s) / d s^{2} \leq 0 \forall s\right)$ or increasing in $s$.

Proposition 3 establishes the optimality of the WTA award scheme under a broad class of distributions for agents' uncertain outputs. To satisfy log-concavity, a density should not be highly convex at any portion of its support (because $\log (h(s))$ is a concave transformation). Many of the commonly-used distributions are log-concave, including Gumbel, exponential, normal, uniform, and logistic distributions. The Weibull distribution can be strictly log-concave, log-linear or strictly log-convex as depicted in Figure 2, but when the Weibull distribution is not log-concave, it is increasing, and hence satisfies condition (7). These conditions can also be interpreted in terms of the primitive technical shock $\widetilde{\epsilon}_{i}$ and taste shock $\widetilde{\varepsilon}_{i}$. Since $\widetilde{\epsilon}_{i}$ has a log-concave density and the convolution of two log-concave distributions is log-concave (e.g., Ibragimov 1956), when the taste shock $\widetilde{\varepsilon}_{i}$ has a log-concave density, the aggregate shock $\widetilde{\xi}_{i}$ also has a log-concave density. For example, when the taste shock $\widetilde{\varepsilon}_{i}$ follows a uniform or normal distribution, the density $h$ of the aggregate shock $\widetilde{\xi}_{i}$ is log-concave as illustrated in Figure 3. Uniform and normal distributions are two popular distributions in modeling symmetric prior beliefs about an unknown value (e.g., 


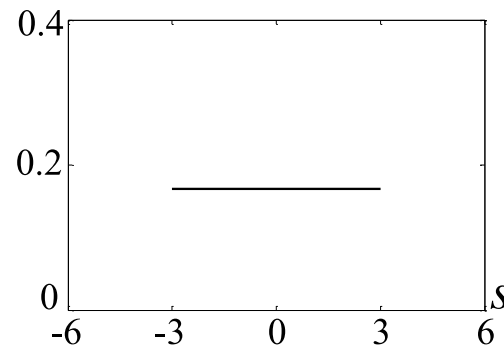

(a)

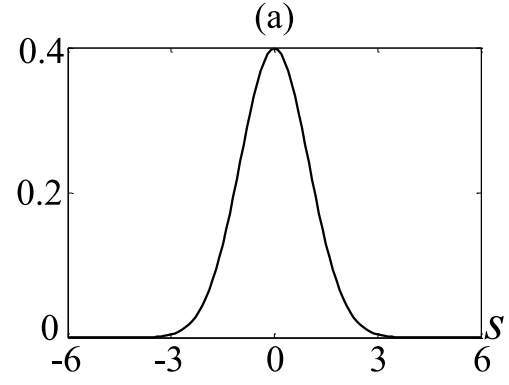

(b)

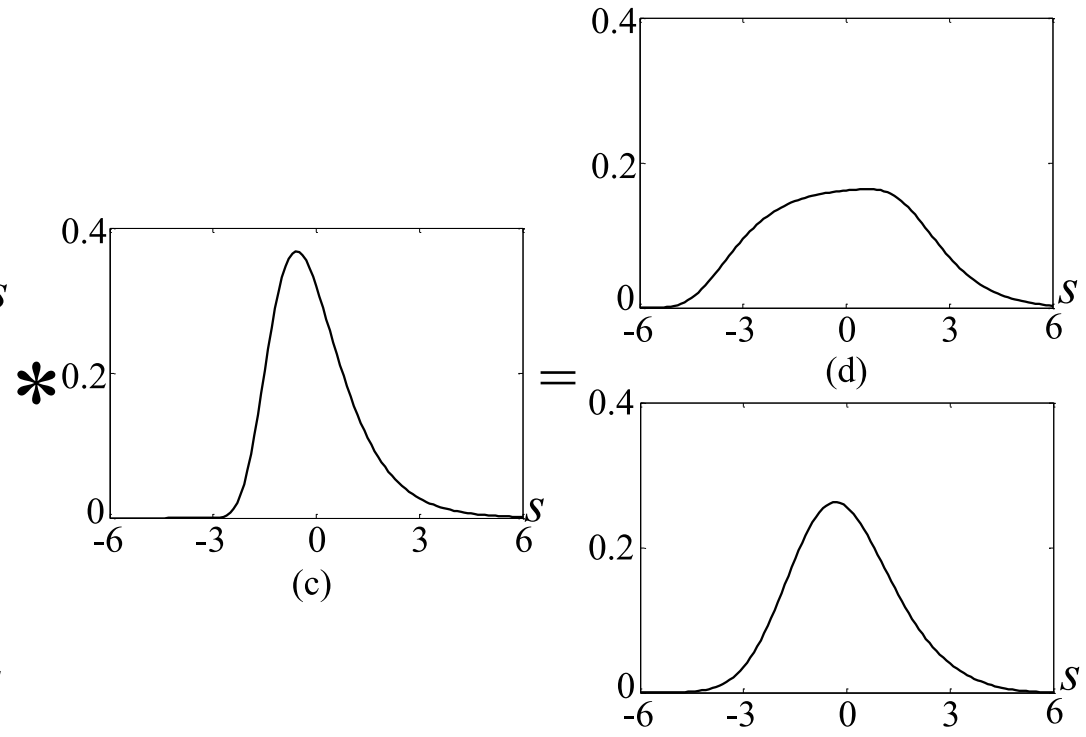

(e)

Figure 3 (a) Uniform density in $[-3,3]$; (b) Normal density with mean 0 and variance 1; (c) Gumbel density with mean 0 and $\mu=1$; (d) The density $h$ of the aggregate output shock $\widetilde{\xi}_{i}$ as the convolution of the densities in (a) and (c); (e) The density $h$ as the convolution of the densities in (b) and (c).

Gelman et al. 2003), and thus they are appropriate for modeling the situation where agents have roughly equal beliefs about the chances of getting more positive or negative evaluations than they deserve for their submitted solutions. In practice, as we discuss in $\S 1$, a number of RTP Challenges at InnoCentive and innovation tournaments at Ennomotive adopt the WTA scheme.

Lastly, we examine the optimal winner prize denoted by $A^{*}$ in a WTA tournament. Observe in the organizer's profit $\Pi_{o}$ given in (4) that the second term $\left(E\left[\sum_{j=1}^{K} \widetilde{\xi}_{(j)}^{N}\right]\right)$ is independent of the winner prize $A$ and the third term $\left(-\sum_{j=1}^{N} A_{(j)}=-A\right)$ decreases with $A$. We can also show that the effort $e^{*}$, hence the first term $\left(K r\left(e^{*}\right)\right)$ in $(4)$, is increasing in $A$. Consequently, the organizer faces the following intuitive trade-off in choosing a value of $A$ : A larger prize motivates agents to exert higher effort, but it costs more to the organizer. In the following proposition, we present a condition under which a unique optimal winner prize $A^{*}$ exists, and show that $A^{*}$ increases with the number of contributors $K$ and it can be increasing, constant or decreasing with the number of participants $N$.

Proposition 4. Let $g(e)=\frac{r^{\prime}(e)}{\psi^{\prime}(e)}$. Suppose that $r(\cdot)$ and $\psi(\cdot)$ satisfy $\frac{g(e) g^{\prime \prime}(e)}{\left(g^{\prime}(e)\right)^{2}} \leq 2$ for all $e \in \mathbb{R}_{+}$.

(a) For any fixed $N$, a unique optimal winner prize $A^{*}$ exists, and it is increasing in $K$.

(b) Suppose $I_{N} \equiv \int_{s \in \Xi} H\left(r(e)-r\left(e^{*}\right)+s\right)^{N-1} h(s) d s$ is decreasing (resp., increasing) in $N$. Then, $A^{*}$ is increasing, constant, or decreasing in $N$ if and only if $\frac{g(e) r^{\prime}(e)}{g^{\prime}(e)}$ (resp., $\left.\frac{g^{\prime}(e)}{g(e) r^{\prime}(e)}\right)$ is increasing, constant, or decreasing in e, respectively. 
The condition on $g$ given in Proposition 4 is a sufficient condition for $\Pi_{o}$ to be concave in $A$; a necessary and sufficient condition is also presented in the proof. In this case, Proposition 4(a) shows, as expected, that the organizer should increase the optimal winner prize $A^{*}$ as he is interested in obtaining a larger number of good solutions from a tournament. Counter-intuitively, however, Proposition 4(b) shows that the optimal prize $A^{*}$ can be increasing, decreasing or constant with the number of participants $N$. To illustrate, consider the following example.

EXAMPLE 1. Suppose the effort function $r(e)=\gamma+\theta \frac{e^{1-a}-1}{1-a}$ and the cost function $\psi(e)=c e$, where $\gamma \in \mathbb{R}, \theta, c>0$, and $a \geq 0.5$. Then, $r^{\prime}(e)=\theta e^{-a}, \psi^{\prime}(e)=c, g(e)=\frac{r^{\prime}(e)}{\psi^{\prime}(e)}=\frac{\theta}{c} e^{-a}, g^{\prime}(e)=-\frac{a \theta}{c} e^{-a-1}$, and hence $\frac{g(e) r^{\prime}(e)}{g^{\prime}(e)}=-\frac{\theta e^{1-a}}{a}$. Also suppose that the output shock $\widetilde{\xi}_{i}$ follows a Gumbel distribution with mean 0 and scale parameter $\mu$. In this case, $I_{N}=\frac{N-1}{\mu N^{2}}$, which is decreasing in $N$. Thus, $A^{*}$ is increasing in $N$ when $a>1$, constant in $N$ when $a=1$, and decreasing in $N$ when $a<1$. Note that $\lim _{a \rightarrow 1} r(e)=\gamma+\theta \log e$, so $A^{*}$ is constant in $N$ when $r(e)$ takes a logarithmic form.

Example 1 includes the special case considered in Terwiesch and $\mathrm{Xu}$ (2008) in which $a=1$, hence optimal prize $A^{*}$ is constant with the number of participants $N$. Alternatively, one might expect that as more participants enter the tournament, the organizer should increase the prize $A$ so as to incentivize an agent to increase effort $e^{*}$. However, Proposition 4(b) shows that this is not necessarily true. The reason is as follows. When more participants reduce the marginal contribution of the prize $A$ to the organizer's profit, the organizer should reduce the prize $A$. From (4), we know this happens when increasing $N$ reduces $\frac{\partial r\left(e^{*}\right)}{\partial A}=r^{\prime}\left(e^{*}\right) \frac{\partial e^{*}}{\partial A}$. If agents reduce their effort with more participants (which happens when $I_{N}$ decreases with $N$ ), then more participants will increase the marginal contribution of effort to the output $r$ (i.e., $r^{\prime}\left(e^{*}\right)$ ), while decreasing the marginal increase of effort with the increased prize (i.e., $\left.\frac{\partial e^{*}}{\partial A}\right)$. In this case, the former effect of more participants is less significant than the latter effect (so that increasing $N$ reduces $\frac{\partial r\left(e^{*}\right)}{\partial A}$ ) when the marginal contribution of effort to output is inelastic to a change in effort (i.e., $\left.\left|\frac{d \log \left(r^{\prime}(e)\right)}{d \log (e)}\right|=-\frac{r^{\prime \prime}(e) e}{r^{\prime}(e)}=a<1\right)$. In practice, this may correspond to situations in which the organizer seeks solutions to a large-scale technical project (e.g., developing autonomous vehicles for DARPA Grand Challenge) rather than a simple ideation project because solving a large-scale technical problem usually requires consistent and significant effort, whereas generating an average-quality idea for a simple problem requires relatively low effort, yet additional effort does not improve the quality of the idea much. In this case, it is optimal for the organizer to reduce the prize $A$ with more participants.

\section{Conclusion}

We have studied an innovation tournament in which an organizer seeks solution(s) to an innovationrelated problem from a number of agents. In order to incentivize agents to exert costly effort in developing good solutions, the organizer should design an award scheme carefully. On the one hand, 
offering a small number of prizes reduces each agent's individual chance of winning a prize. This may discourage agents from exerting their best effort or even from participating in the tournament. On the other hand, offering too many prizes may reduce agents' effort because agents could receive a prize even with low effort. In this paper, we provide useful insights into selecting the number and amount of awards.

By analyzing a general model that takes into account uncertainty in both developing and evaluating innovative solutions, we generate the following insights. First, it may be optimal for the organizer to offer multiple prizes when agents perceive it very likely that only few agents receive high evaluation while most of them receive low evaluation (e.g., tournaments wherein submitted solutions are evaluated based on the popularity among consumers) or when a tournament does not require substantial increase in agents' marginal cost of effort to develop high-quality solutions as in ideation contests. Second, it is optimal for the organizer to offer one large prize to the winner than multiple small prizes in many practical situations, especially when agents have symmetric beliefs about the organizer's taste. In this case, the organizer should offer a larger winner prize when he is interested in obtaining a higher number of good solutions, but the organizer need not necessarily raise the winner prize when anticipating more participants to a tournament. Given the limited results in prior literature and mixed observations in practice, these insights into the optimal award scheme provide useful guidance to practitioners who organize innovation tournaments.

There are interesting future research avenues. First, while our model assumes that $N$ is common knowledge, in practice, all parties in the tournament may not have perfect information about $N$. Our main results extend to the case where the organizer and agents have a common distribution for unknown $N$. It would be interesting to analyze the case where an organizer and agents have asymmetric beliefs about unknown $N$. Second, while we have considered the case wherein the organizer awards agents based on their relative ranks, there are other compensation rules that the organizer may use such as awarding agents based on their absolute performance rather than relative ranks. An interesting future research direction would be to compare the organizer's profit under different compensation rules, considering the impact of the number of solutions that the organizer seeks and the uncertainty agents face. This analysis can be enriched by comparing different tournament formats or by incorporating behavioral effects.

\section{Appendix}

Proof of Lemma 1. Let $\widetilde{\epsilon}_{i,(1)}^{m_{i}}=\max \left\{\widetilde{\epsilon}_{i t}, t=1,2, \ldots, m_{i}\right\}$. Then, the output function $y$ in (1) can be written as $y\left(q_{i}, m_{i}, \widetilde{\epsilon}_{i,(1)}^{m_{i}}, \widetilde{\varepsilon}_{i}\right)=v\left(q_{i}\right)+\widetilde{\epsilon}_{i,(1)}^{m_{i}}+\widetilde{\varepsilon}_{i}$. Since $\widetilde{\epsilon}_{i t}$ follows Gumbel distribution with mean zero and scale parameter $\mu$, by the property given in Dahan and Mendelson (2001) (pg 115, Appendix B), $\widetilde{\epsilon}_{i,(1)}^{m_{i}}$ follows a Gumbel distribution with mean $\mu \log m_{i}$ and scale parameter $\mu$. Furthermore, 
$\widetilde{\epsilon}_{i}=\widetilde{\epsilon}_{i,(1)}^{m_{i}}-\mu \log m_{i}$ follows a Gumbel distribution with mean 0 and scale parameter $\mu$. Thus, the output function simplifies to $y\left(q_{i}, m_{i}, \widetilde{\epsilon}_{i}, \widetilde{\varepsilon}_{i}\right)=v\left(q_{i}\right)+\mu \log m_{i}+\widetilde{\epsilon}_{i}+\widetilde{\varepsilon}_{i}$. Given total effort $e_{i}$, agent $i$ can choose $q_{i}$ and $m_{i}$ to maximize her output by solving:

$$
r\left(e_{i}\right)=\max _{q_{i}, m_{i}} v\left(q_{i}\right)+\mu \log m_{i} \quad \text { s.t. } \quad c_{1} q_{i}+c_{2} m_{i}=e_{i} .
$$

It is not difficult to verify that $r\left(e_{i}\right)$ is concave and increasing in $e_{i}$. Therefore, the agent's output function simplifies to $y\left(e_{i}, \widetilde{\xi}_{i}\right)=r\left(e_{i}\right)+\widetilde{\xi}_{i}$, where $\widetilde{\xi}_{i}=\widetilde{\epsilon}_{i}+\widetilde{\varepsilon}_{i}$.

Suppose that $v\left(q_{i}\right)=\kappa \log q_{i}$. Then, (9) yields $q_{i}^{*}=\frac{\kappa e_{i}}{c_{1}(\kappa+\mu)}$ and $m_{i}^{*}=\frac{\mu e_{i}}{c_{2}(\kappa+\mu)}$. Thus, $r\left(e_{i}\right)=$ $\kappa \log q_{i}^{*}+\mu \log m_{i}^{*}=\gamma+\theta \log e_{i}$, where $\theta=\kappa+\mu$ and $\gamma=\kappa \log \frac{\kappa}{c_{1}(\kappa+\mu)}+\mu \log \frac{\kappa}{c_{2}(\kappa+\mu)}$.

Proof of Proposition 1. Suppose that (7) is satisfied, but the WTA scheme is not optimal. Then, there exists $l(>1)$ such that $A_{(l)}>0$ in an optimal award scheme. Let $e$ be the equilibrium effort corresponding to this award scheme. Consider the following perturbation where the $l$-th prize is shifted to the winner prize; i.e., let $\widehat{A}_{(l)}=A_{(l)}-A_{(l)}=0$ and $\widehat{A}_{(1)}=A_{(1)}+A_{(l)}$, while all other $A_{(j)}$ 's remain unchanged. Denote with $\widehat{e}$ the corresponding equilibrium effort. Since the total prize $A$ remains the same, the perturbation weakly improves $\Pi_{o}$ if agents exercise weakly higher effort under this new award scheme (i.e., $\widehat{e} \geq e)$. By (7), $\frac{\partial P_{(1)}^{N}}{\partial e_{i}}[e, e] \geq \frac{\partial P_{(j)}^{N}}{\partial e_{i}}[e, e]$ for all $j>1$. Thus,

$$
\sum_{j=1}^{N} \frac{\partial P_{(j)}^{N}}{\partial e_{i}}[e, e] \widehat{A}_{(j)} \geq \sum_{j=1}^{N} \frac{\partial P_{(j)}^{N}}{\partial e_{i}}[e, e] A_{(j)}=\psi^{\prime}(e),
$$

where the last equation follows from the fact that $e$ satisfies the first-order condition of (6). The inequality in (10) implies that after the perturbation, an agent will not have lower effort. Applying this perturbation for all $l(>1)$ such that $A_{(l)}>0$, we find that the organizer's utility under the WTA is at least as good as that under the optimal award scheme, which is a contradiction.

Next, suppose that the WTA scheme is optimal. Let $e$ be the corresponding effort in equilibrium. Suppose to the contrary that $e$ violates $(7)$. Then, there exists $l(>1)$ such that $\frac{\partial P_{(1)}^{N}}{\partial e_{i}}[e, e]<$ $\frac{\partial P_{(l)}^{N}}{\partial e_{i}}[e, e]$. Similar to the perturbation above, let $\widehat{A}_{(l)}=\delta$ and $\widehat{A}_{1}=A_{1}-\delta$ with small $\delta>0$. This perturbation improves agents' effort, and hence contradicts the optimality of the WTA scheme.

Proof of Proposition 2. To simplify the notation, let $P_{(j)}^{N}[e] \equiv P_{(j)}^{N}\left[e, e^{*}\right]$. From (3), we can verify

$$
P_{(j)}^{N}[e]=\int_{\underline{s}}^{\bar{s}} W_{(j)}^{N}\left(s+r(e)-r\left(e^{*}\right)\right) h(s) d s, \text { where } W_{(j)}^{N}(s)=\frac{(N-1) !}{(j-1) !(N-j) !} H(s)^{N-j}(1-H(s))^{j-1}
$$

Taking the derivative of $P_{(j)}^{N}[e]$ with respect to $e$, evaluating it at $e^{*}$, and using integration by parts,

$$
\frac{\partial P_{(j)}^{N}}{\partial e}\left[e^{*}\right]=r^{\prime}\left(e^{*}\right) \int_{\underline{s}}^{\bar{s}}\left(W_{(j)}^{N}\right)^{\prime}(s) h(s) d s=r^{\prime}\left(e^{*}\right) \lim _{s \rightarrow \bar{s}} W_{(j)}^{N}(s) h(s)-r^{\prime}\left(e^{*}\right) \int_{\underline{s}}^{\bar{s}} W_{(j)}^{N}(s) h^{\prime}(s) d s .
$$

Here $\lim _{s \rightarrow \bar{s}} W_{(j)}^{N}(s) h(s)=\lim _{s \rightarrow \bar{s}} \frac{h_{(j)}^{N}(s)}{N}$, where $h_{(j)}^{N}(s)=\frac{N !}{(j-1) !(N-j) !} H(s)^{N-j}(1-H(s))^{j-1} h(s)$. Note that $\lim _{s \rightarrow \bar{s}} h_{(j)}^{N}(s) / N=0$ for any $j \in\{2, \ldots, N\}$ and $\lim _{s \rightarrow \bar{s}} h_{(1)}^{N}(s) / N=w_{1} \geq 0$. Thus, $\frac{\partial P_{(1)}^{N}}{\partial e}\left[e^{*}\right]-\frac{\partial P_{(j)}^{N}}{\partial e}\left[e^{*}\right]=w_{1}+r^{\prime}\left(e^{*}\right) \int_{\underline{s}}^{\bar{s}}\left[W_{(j)}^{N}(s)-W_{(1)}^{N}(s)\right] h^{\prime}(s) d s=w_{1}+\frac{r^{\prime}\left(e^{*}\right)}{N} \int_{\underline{s}}^{\bar{s}}\left[h_{(j)}^{N}(s)-h_{(1)}^{N}(s)\right] \frac{h^{\prime}(s)}{h(s)} d s$, 
for any $j \in\{2, \ldots, N\}$. Using integration by parts, the expression above boils down to

$$
\frac{\partial P_{(1)}^{N}}{\partial e}\left[e^{*}\right]-\frac{\partial P_{(j)}^{N}}{\partial e}\left[e^{*}\right]=w_{1}+\frac{r^{\prime}\left(e^{*}\right)}{N}\left(\lim _{s \rightarrow \bar{s}}\left[H_{(j)}^{N}(s)-H_{(1)}^{N}(s)\right] \frac{h^{\prime}(s)}{h(s)}+\int_{\underline{s}}^{\bar{s}}\left[H_{(j)}^{N}(s)-H_{(1)}^{N}(s)\right]\left(\frac{h^{\prime}(s)}{h(s)}\right)^{\prime} d s\right) .
$$

Suppose that $h$ satisfies condition (i). Then in (11), $w_{1}=0$ since $\lim _{s \rightarrow \bar{s}} h(s)=0$, and also $\lim _{s \rightarrow \bar{s}}\left[H_{(j)}^{N}(s)-H_{(1)}^{N}(s)\right] \frac{h^{\prime}(s)}{h(s)}=0$ because $\lim _{s \rightarrow \bar{s}}\left|\frac{h^{\prime}(s)}{h(s)}\right|<\infty$. Thus, by $(8), \frac{\partial P_{(1)}^{N}}{\partial e}\left[e^{*}\right]-\frac{\partial P_{(j)}^{N}}{\partial e}\left[e^{*}\right]<0$, and hence the WTA scheme is not optimal. When $h$ is log-convex, $\left(\frac{h^{\prime}(s)}{h(s)}\right)^{\prime}>0$ for all $s$ so (8) is satisfied, noting that $H_{(j)}^{N}(s)-H_{(1)}^{N}(s) \geq 0$ for all $s$ (>0 for a measurable subset of $\left.\Xi\right)$.

Suppose that condition (ii) is satisfied. The solution to (6) under the WTA scheme, $e^{*}=$ $\left(\frac{\psi^{\prime}}{r^{\prime}}\right)^{-1}\left(A \int_{s \in \Xi}(N-1) H(s)^{N-2} h(s)^{2} d s\right)$, violates $(5)$ because $\frac{A}{N}-\psi\left(e^{*}\right)<0$. Thus, the WTA scheme is not feasible for the organizer's problem, and hence it is not optimal.

Proof of Proposition 3. We will show that when $h$ is log-concave, $\frac{\partial P_{(1)}^{N}}{\partial e}\left[e^{*}\right] \geq \frac{\partial P_{(j)}^{N}}{\partial e}\left[e^{*}\right]$ for all $j \in$ $\{2, \ldots, N\}$. When $h$ is log-concave, in $(11), \lim _{s \rightarrow \bar{s}}\left[H_{(j)}^{N}(s)-H_{(1)}^{N}(s)\right] \frac{h^{\prime}(s)}{h(s)}=0$. The term $H_{(j)}^{N}(s)-$ $H_{(1)}^{N}(s) \geq 0$ for all $s$ (>0 for a measurable subset of $\Xi$ ) since $\xi_{(1)}^{N}$ first-order stochastically dominates $\xi_{(j)}^{N}$. Therefore, a sufficient condition for (11) to be positive is that $\left(\frac{h^{\prime}(s)}{h(s)}\right)^{\prime} \leq 0$ for all $s$. This is indeed the case when $h$ is log-concave.

Next, suppose that $h$ is increasing. We will show that $\frac{\partial P_{(1)}^{N}}{\partial e}\left[e^{*}\right] \geq \frac{\partial P_{(j)}^{N}}{\partial e}\left[e^{*}\right]$ for any $j>1$. Observe that $\frac{\partial P_{(j)}^{N}}{\partial e}\left[e^{*}\right]=r^{\prime}\left(e^{*}\right) \int_{\underline{s}}^{\bar{s}}\left(W_{(j)}^{N}\right)^{\prime}(s) h(s) d s$, where $\left(W_{(1)}^{N}\right)^{\prime}=h_{(1)}^{N-1}(s),\left(W_{(N)}^{N}\right)^{\prime}=-h_{(N-1)}^{N-1}(s)$, and $\left(W_{(j)}^{N}\right)^{\prime}(s)=\frac{(N-1) !}{(j-1) !(N-j) !}\left[(N-j) H(s)^{N-j-1}(1-H(s))^{j-1}-(j-1) H(s)^{N-j}(1-H(s))^{j-2}\right] h(s)$, for $j \in\{2, \ldots, N-1\}$. Then $\left.\left(W_{(j)}^{N}\right)^{\prime}(s)=h_{(j)}^{N-1}(s)-h_{(j-1)}^{N-1}(s)\right)$, and so $\frac{\partial P_{(1)}^{N}}{\partial e}\left[e^{*}\right]=r^{\prime}\left(e^{*}\right) E\left[h\left(\widetilde{\xi}_{(1)}^{N-1}\right)\right]$,

$$
\frac{\partial P_{(j)}^{N}}{\partial e}\left[e^{*}\right]=r^{\prime}\left(e^{*}\right) \int_{\underline{s}}^{\bar{s}}\left[h_{(j)}^{N-1}(s)-h_{(j-1)}^{N-1}(s)\right] h(s) d s=r^{\prime}\left(e^{*}\right)\left(E\left[h\left(\widetilde{\xi}_{(j)}^{N-1}\right)\right]-E\left[h\left(\widetilde{\xi}_{(j-1)}^{N-1}\right)\right]\right),
$$

for $j \in\{2, \ldots, N-1\}$, and $\frac{\partial P_{(N)}^{N}}{\partial e}\left[e^{*}\right]=-r^{\prime}\left(e^{*}\right) E\left[h\left(\widetilde{\xi}_{(N-1)}^{N-1}\right)\right]$. When $h$ is increasing, $E\left[h\left(\widetilde{\xi}_{(1)}^{N-1}\right)\right] \geq$ $E\left[h\left(\widetilde{\xi}_{(j)}^{N-1}\right)\right]$ for any $j>1$ because $\widetilde{\xi}_{(1)}^{N-1}$ first-order stochastically dominates $\widetilde{\xi}_{(j)}^{N-1}$. Moreover, $E\left[h\left(\widetilde{\xi}_{(j)}^{N-1}\right)\right] \geq 0$ for any $j$ because $h \geq 0$. Therefore, $\frac{\partial P_{(1)}^{N}}{\partial e}\left[e^{*}\right] \geq \frac{\partial P_{(j)}^{N}}{\partial e}\left[e^{*}\right]$ for any $j \in\{2, \ldots, N\}$.

Remark. Note that (7) is independent of $e^{*}$ because letting $W_{(j)}^{N}=\frac{h_{(j)}^{N}(s)}{N h(s)}$, we have for all $j>1$,

$$
\begin{aligned}
\left.\frac{\partial P_{(1)}^{N}\left[e, e^{*}\right]}{\partial e}\right|_{e=e^{*}} \geq\left.\frac{\partial P_{(j)}^{N}\left[e, e^{*}\right]}{\partial e}\right|_{e=e^{*}} & \Longleftrightarrow r^{\prime}\left(e^{*}\right) \int_{\underline{s}}^{\bar{s}}\left(W_{(1)}^{N}\right)^{\prime}(s) h(s) d s \geq r^{\prime}\left(e^{*}\right) \int_{\underline{s}}^{\bar{s}}\left(W_{(j)}^{N}\right)^{\prime}(s) h(s) d s \\
& \Longleftrightarrow \int_{\underline{s}}^{\bar{s}}\left(W_{(1)}^{N}\right)^{\prime}(s) h(s) d s \geq \int_{\underline{s}}^{\bar{s}}\left(W_{(j)}^{N}\right)^{\prime}(s) h(s) d s .
\end{aligned}
$$

Proof of Proposition 4. We first derive a necessary and sufficient condition for the organizer's profit $\Pi_{o}$ to be concave in the winner prize $A$, and then show that the assumption in Proposition 4 is sufficient for concavity. From (4), $\frac{\partial^{2} \Pi_{o}}{\partial A^{2}}=K r^{\prime \prime}\left(e^{*}\right)\left(\frac{\partial e^{*}}{\partial A}\right)^{2}+K r^{\prime}\left(e^{*}\right) \frac{\partial^{2} e^{*}}{\partial A^{2}}$. Thus, we need to characterize the equilibrium effort $e^{*}$. Under the WTA, the agent's problem in (6) becomes

$$
\max _{e \in \mathbb{R}_{+}} A P_{(1)}^{N}\left[e, e^{*}\right]-\psi(e)=\max _{e \in \mathbb{R}_{+}} A \int_{s \in \Xi} H\left(r(e)-r\left(e^{*}\right)+s\right)^{N-1} h(s) d s-\psi(e) .
$$


Substituting $e=e^{*}$ into the first order condition of (12) yields

$$
\frac{\psi^{\prime}\left(e^{*}\right)}{r^{\prime}\left(e^{*}\right)}=A \int_{s \in \Xi}(N-1) H(s)^{N-2} h(s)^{2} d s .
$$

From (13), the equilibrium effort is $e^{*}=g^{-1}\left(1 /\left(A I_{N}\right)\right)$, where $g=\frac{r^{\prime}}{\psi^{\prime}}$ and $I_{N}=$ $\int_{s \in \Xi}(N-1) H(s)^{N-2} h(s)^{2} d s$. This implies that $\frac{\partial e^{*}}{\partial A}=-\frac{1}{g^{\prime}\left(e^{*}\right)} \frac{1}{A^{2} I_{N}}=-\frac{g\left(e^{*}\right)}{A g^{\prime}\left(e^{*}\right)}$, and hence $\frac{\partial^{2} e^{*}}{\partial A^{2}}=$ $\frac{1}{A} \frac{\partial e^{*}}{\partial A}\left[-2+\frac{g\left(e^{*}\right) g^{\prime \prime}\left(e^{*}\right)}{\left(g^{\prime}\left(e^{*}\right)\right)^{2}}\right]$. Thus, $\Pi_{o}$ is concave in $A$ if and only if

$$
\frac{\partial^{2} \Pi_{o}}{\partial A^{2}}=\frac{1}{A} \frac{\partial e^{*}}{\partial A}\left[r^{\prime}\left(e^{*}\right)\left(-2+\frac{g\left(e^{*}\right) g^{\prime \prime}\left(e^{*}\right)}{\left(g^{\prime}\left(e^{*}\right)\right)^{2}}\right)-r^{\prime \prime}\left(e^{*}\right) \frac{g\left(e^{*}\right)}{A g^{\prime}\left(e^{*}\right)}\right] \leq 0 .
$$

Since $r$ is concave and increasing in $e$, and $\psi$ is convex and increasing in $e$, we have $r^{\prime}>0, r^{\prime \prime} \leq 0$, $g>0$, and $g^{\prime} \leq 0$. Furthermore, by the assumption, $-2+\frac{g\left(e^{*}\right) g^{\prime \prime}\left(e^{*}\right)}{\left(g^{\prime}\left(e^{*}\right)\right)^{2}} \leq 0$. Therefore, $\frac{\partial^{2} \Pi_{o}}{\partial A^{2}} \leq 0$.

(a) Due to concavity of $\Pi_{o}$ with respect to $A$, the optimal prize $A^{*}[K]$ when there are $K$ contributors satisfies the first order condition of the organizer's problem with respect to $A$, i.e.,

$$
\frac{\partial \Pi_{o}\left(A^{*}[K]\right)}{\partial A}=\left.K r^{\prime}\left(e^{*}\right) \frac{\partial e^{*}}{\partial A}\right|_{A=A^{*}[K]}-1=0 .
$$

Suppose that $K$ increases to $K+1$. Suppose to the contrary that $A^{*}[K+1] \leq A^{*}[K]$ (i.e., $A^{*}$ is nonincreasing in $K)$. Observe from (4) that $\Pi_{o}$ is concave in $A$ if and only if $r\left(e^{*}\right)$ is concave in $A$, which implies that $r^{\prime}\left(e^{*}\right) \frac{\partial e^{*}}{\partial A}$ is non-increasing in $A$. Then, $\left.r^{\prime}\left(e^{*}\right) \frac{\partial e^{*}}{\partial A}\right|_{A=A^{*}[K+1]} \geq\left. r^{\prime}\left(e^{*}\right) \frac{\partial e^{*}}{\partial A}\right|_{A=A^{*}[K]}$ since $A^{*}[K+1] \leq A^{*}[K]$. However, in this case, $\left.(K+1) r^{\prime}\left(e^{*}\right) \frac{\partial e^{*}}{\partial A}\right|_{A=A^{*}[K+1]}>\left.K r^{\prime}\left(e^{*}\right) \frac{\partial e^{*}}{\partial A}\right|_{A=A^{*}[K]}=1$, which implies that $A^{*}[K+1]$ violates (15). Therefore, $A^{*}[K+1]>A^{*}[K]$.

(b) Let $\Phi=\left.K r^{\prime}\left(e^{*}\right) \frac{\partial e^{*}}{\partial A}\right|_{A=A}$. From our derivation in part (a), $\frac{\partial e^{*}}{\partial A}=-\frac{g\left(e^{*}\right)}{A g^{\prime}\left(e^{*}\right)}$, so

$$
\Phi=-K r^{\prime}\left(e^{*}\right) \frac{g\left(e^{*}\right)}{A^{*} g^{\prime}\left(e^{*}\right)}=-K r^{\prime}\left(g^{-1}\left(1 / A^{*} I_{N}\right)\right) \frac{g\left(g^{-1}\left(1 / A^{*} I_{N}\right)\right)}{A^{*} g^{\prime}\left(g^{-1}\left(1 / A^{*} I_{N}\right)\right)}=0 .
$$

By applying Implicit Function Theorem to (16), we obtain: $\frac{\partial A^{*}}{\partial N}=-\frac{\frac{\partial \Phi}{\partial N}}{\frac{\partial \Phi}{\partial A^{*}}}$. As we show in part (a), (14) holds so $\frac{\partial \Phi}{\partial A^{*}} \leq 0$. Thus, $\frac{\partial A^{*}}{\partial N}$ has the same sign as $\frac{\partial \Phi}{\partial N}$. Noting that $e^{*}=g^{-1}\left(1 /\left(A I_{N}\right)\right)$,

$$
\frac{\partial \Phi}{\partial N}=\frac{\partial}{\partial e^{*}}\left[r^{\prime}\left(e^{*}\right) \frac{g\left(e^{*}\right)}{g^{\prime}\left(e^{*}\right)}\right] \frac{K}{A^{*} g^{\prime}\left(e^{*}\right)} \frac{1}{A^{*}\left(I_{N}\right)^{2}} \frac{\partial I_{N}}{\partial N} .
$$

Because $g^{\prime} \leq 0, K>0, A^{*}>0$, and $\left(1 /\left(A^{*}\left(I_{N}\right)^{2}\right)>0\right.$, the derivative $\frac{\partial \Phi}{\partial N}$ has the opposite sign of $\frac{\partial}{\partial e^{*}}\left[r^{\prime}\left(e^{*}\right) \frac{g\left(e^{*}\right)}{g^{\prime}\left(e^{*}\right)}\right] \frac{\partial I_{N}}{\partial N}$. Suppose that $\frac{\partial I_{N}}{\partial N}<0$. Then, $A^{*}$ is increasing, constant, or decreasing in $N$ if and only if $\frac{g(e) r^{\prime}(e)}{g^{\prime}(e)}$ is increasing, constant, or decreasing in $N$, respectively. Suppose that $\frac{\partial I_{N}}{\partial N}>0$. Note that $\frac{g(e) r^{\prime}(e)}{g^{\prime}(e)}$ is increasing whenever $\frac{g^{\prime}(e)}{g(e) r^{\prime}(e)}$ is decreasing. Thus, $A^{*}$ is increasing, constant, or decreasing in $N$ if and only if $\frac{g^{\prime}(e)}{g(e) r^{\prime}(e)}$ is increasing, constant, or decreasing in $N$, respectively.

\section{Endnotes}

1. In Lemma EC.1 of Online Appendix, we show that the WTA award scheme may not be optimal even if it yields a higher profit to the organizer than awarding the winner and/or the runner-up. Under deterministic settings, Moldovanu and Sela (2001) show that the WTA scheme is optimal when the agents' cost functions of effort are linear or concave, but may not be optimal when the cost functions are convex. 
2. Here we assume that the number of agents $N$ is common knowledge. This is the common assumption used by all previous papers in the literature reviewed in $\S 1$. In practice, although all parties may not have perfect information about $N$, there are often mechanisms that could allow an organizer and agents to get a good estimate of $N$. For example, InnoCentive and Ennomotive publish the number of participants in each tournament, and DARPA holds a Competitors Conference to gauge the number of participants. Our main results extend to the case when $N$ is unknown with some distribution although expressions for conditions become more complex.

3. This model takes two popular approaches in the literature as special cases: $K=1$ (e.g., Taylor 1995, Che and Gale 2003) and $K=N$ (e.g., Green and Stokey 1983, Kalra and Shi 2001). It further considers the most practically-common cases of $K \in(1, N)$.

4. Note that $N$ is the number of agents who can participate in a tournament, and that $N$ becomes the number of participants when participation constraint (5) is satisfied. This is the standard approach to analyzing an award scheme in the extant literature (Moldovanu and Sela 2001, Kalra and Shi 2001, Terwiesch and Xu 2008). Ales et al. (2016) and Korpeoglu and Cho (2015) further study when it is optimal for the organizer to have an open tournament with free entry or to restrict entry under the WTA scheme. In a free-entry open tournament which allows all agents who wish to participate to do so, $N$ corresponds to a pool of agents who can potentially participate in the tournament. If the organizer restricts entry to the tournament, $N$ corresponds to the number of agents that are allowed by the organizer to enter.

5. $\lim _{s \rightarrow \bar{s}} h(s)=0$ when $\bar{s}=\infty$ and the density $h$ of $\widetilde{\xi}_{i}$ is decreasing in $s$ as $s$ approaches $\bar{s}$. $\lim _{s \rightarrow \bar{s}}\left|h^{\prime}(s) / h(s)\right|<\infty$ for gamma, lognormal and Frechet distributions. Likewise, $\lim _{s \rightarrow \bar{s}}\left|h^{\prime}(s) / h(s)\right|<\infty$ for Pareto distribution, and similar fat-tailed distributions that satisfy $h(s) \sim s^{-(1+\gamma)}$ and $h^{\prime}(s) \sim-(1+\gamma) s^{-(2+\gamma)}$ for some $\gamma>0$ as $s \rightarrow \infty$.

6. Let $s_{(j)}$ be such that $h\left(s_{(j)}\right)=E\left[h\left(\widetilde{\xi}_{(j)}^{N-1}\right)\right]$ (i.e., $s_{(j)}$ represents the certainty equivalent of $\widetilde{\xi}_{(j)}^{N-1}$ under the density $\left.h\right)$. In the proof of Proposition 3, we show that $\frac{\partial P_{(1)}^{N}\left[e^{*}, e^{*}\right]}{\partial e}=r^{\prime}\left(e^{*}\right) h\left(s_{(1)}\right)$ and $\frac{\partial P_{(2)}^{N}\left[e^{*}, e^{*}\right]}{\partial e}=r^{\prime}\left(e^{*}\right)\left\{h\left(s_{(2)}\right)-h\left(s_{(1)}\right)\right\}$. Figure 1(c) illustrates that in this example, $\frac{\partial P_{(1)}^{N}\left[e^{*}, e^{*}\right]}{\partial e}=$ $r^{\prime}\left(e^{*}\right) h\left(s_{(1)}\right)=0.065 r^{\prime}\left(e^{*}\right)$ and $\frac{\partial P_{(2)}^{N}\left[e^{*}, e^{*}\right]}{\partial e}=r^{\prime}\left(e^{*}\right)\left\{h\left(s_{(2)}\right)-h\left(s_{(1)}\right)\right\}=(0.133-0.065) r^{\prime}\left(e^{*}\right)=$ $0.068 r^{\prime}\left(e^{*}\right)$. Thus, the highly convex and decreasing region of $h(s)$ leads to $\frac{\partial P_{(1)}^{N}\left[e^{*}, e^{*}\right]}{\partial e}<\frac{\partial P_{(2)}^{N}\left[e^{*}, e^{*}\right]}{\partial e}$.

\section{Acknowledgments}

We appreciate helpful comments from seminar participants at Carnegie Mellon University, Duke University, Harvard University, Massachusetts Institute of Technology, University College London, INFORMS Annual Meeting, POM Annual Conference, and MSOM Conference. We also thank the area editor Chung Piaw Teo and his review team for their guidance and constructive comments. This work is partially supported by the Carnegie Bosch Research Grant, Carnegie Mellon University. 


\section{References}

Ales, L., S. Cho, E. Korpeoglu. 2016. Innovation tournaments with multiple contributors. Working Paper, Carnegie Mellon University.

Cha, M., M. Kwak, P. Rodriguez, Y.-Y. Ahn, S. Moon. 2007. I tube, you tube, everybody tubes: Analyzing the world's largest user generated content video system. Proc. of the 7th ACM SIGCOMM Conference on Internet Measurement (IMC 07), San Diego, CA.

Che, Y. K., I. Gale. 2003. Optimal design of research contests. Amer. Econ. Rev. 93(3) 646-671.

Dahan, E., H. Mendelson. 2001. An extreme-value model of concept testing. Manage. Sci. 47(1) 102 - 116.

Ennomotive. 2016. Alternative to shot blasting for aluminum injection parts manufacturing. URL http: //www.ennomotive.com/alternative-shot-blasting-aluminum-injection-parts-10-000-e-prizes/.

Fullerton, R. L., R. P. McAfee. 1999. Auctioning entry into tournaments. J. Polit. Econ. 107(3) 573-605.

Gelman, A., J. B. Carlin, H. S. Stern, D. B. Rubin. 2003. Bayesian Data Analysis. Chapman \& Hall / CRC, Boca Raton, FL.

Green, J. R., N. L. Stokey. 1983. A comparison of tournaments and contracts. J. Polit. Econ. 91(3) 349-364.

Ibragimov, I. A. 1956. On the composition of unimodal distributions. Theor. Probab. Appl. 1 255-260.

InnoCentive. 2016a. New translational models of psychiatric diseases. URL https://www.innocentive.com/ ar/challenge/9933822.

InnoCentive. 2016b. Novo nordisk challenge: Design and synthesis of a small molecule glucose binder. URL https://www.innocentive.com/ar/challenge/9933823.

Kalra, A., M. Shi. 2001. Designing optimal sales contests: a theoretical perspective. Market. Sci. 20(2) 170-193.

Kim, K., M. K. Lim. 2015. R\&D outsourcing in an innovation-driven supply chain. Operations Research Letters 43(1) 20-25.

Korpeoglu, E., S. Cho. 2015. Incentives in contests with heterogeneous solvers. Working Paper, Carnegie Mellon University.

Li, X., L. M. Hitt. 2008. Self-selection and information role of online product reviews. Information Systems Research 19(4) 456-474.

Moldovanu, B., A. Sela. 2001. The optimal allocation of prizes in contests. Amer. Econ. Rev. 91(3) 542-558.

Rothschild, M., J.E. Stiglitz. 1970. Increasing risk: I. a definition. J. Econ. theory 2(3) 225-243.

Samsung. 2012. Winners of samsung smart app challenge 2012 unveiled. URL http://developer.samsung. com/what-is-new/news/2012/Winners-of-Samsung-Smart-App-Challenge-2012-Unveiled.

Taylor, C. R. 1995. Digging for golden carrots: an analysis of research tournaments. Amer. Econ. Rev. 85(4) $872-890$. 
Terwiesch, C., Y. Xu. 2008. Innovation contests, open innovation and multiagent problem solving. Manage. Sci. 54(9) 1529-1543.

Laurence Ales is an associate professor of economics at Tepper School of Business, Carnegie Mellon University. His research interests include optimal contracts, taxation of top incomes, and health and consumption inequality.

Soo-Haeng Cho is an associate professor of operations management at Tepper School of Business, Carnegie Mellon University. His research interests include crowdsourcing and innovation, healthcare supply chains, socially responsible operations, and competition and cooperation in supply chains.

Ersin Körpeoğlu is an assistant professor of operations \& technology at University College London, School of Management. His research interests include crowdsourcing and innovation, competition in supply chains, and business analytics applications to healthcare and scheduling. 